\title{
Extended Release Flucytosine
}

National Cancer Institute

\section{Source}

National Cancer Institute. Extended Release Flucytosine. NCI Thesaurus. Code C112498.

An extended release (ER) oral tablet that contains flucytosine (5-FC), a fluorinated cytosine analog, with antifung al activity and potential anti-cancer activity. Following oral administration of ER 5-FC, the 5-FC is deaminated to its active metabolite 5-fluorouracil (5-FU). 5-FU replaces uracil during RNA synthesis, which consequently inhibits downstream protein synthesis. In addition, 5-FU is metabolized further to 5fluorodeoxyuridylic acid monophosphate, which inhibits thymidylate synthetase. Inhibition of this enzyme interrupts nucleotide synthesis, DNA replication and cell proliferation. Negative regulation of protein synthesis, DNA replication and cell proliferation can lead to cell death. Following ingestion of ER 5-FC, intravenous injection of a retroviral vector encoding cytosine deaminase (TC 511) at a tumor site may result in higher local concentrations of 5-FU and its metabolites, and increased tumor cell death than other 5FU treatment regimens. 